\title{
REVIEW
}

\section{Exosome-mediated immune regulation and its clinical application}

\author{
Naohiro Seo \\ Department of Immuno-Gene Therapy, Mie University Graduate School of Medicine, Japan
}

\begin{abstract}
Immune system is a precise mechanism for maintenance of homeostasis by lymphocyte-mediated elimination of extracellular and intercellular pathogens, and abnormal cells in cytokine-, chemokine-, antibody-, and cytotoxic granuledependent manners. Extracellular vesicles, e.g. exosomes, released from multivesicular endosome in immune cells have been known to be a part of the immune system. Exosomes released by antigen-presenting cells (APCs) such as macrophages and dendritic cells (DCs) regulate natural killer (NK) cells, CD8 ${ }^{+} \mathrm{T}$ cells (Cytotoxic T lymphocytes [CTLs]), and $\mathrm{CD}^{+} \mathrm{T}$ cells (Th cells) including Th1, Th2, and regulatory $\mathrm{T}$ (Treg) cells. In the anti-tumor immune system, NK cells and CTLs are mainly involved in the elimination of tumor cells by direct interaction. Recently, we clarified that tumor-infiltrating $\mathrm{CD} 8^{+} \mathrm{T}$ cells prevent tumor invasion and metastasis by exosome-mediated destruction of tumor stroma consist of mesenchymal stem cells (MSCs) and cancer-associated fibroblasts (CAFs). In this review article, we describe the role of exosomes in controlling immune system and its clinical application.
\end{abstract}

Keywords: $C D 8^{+} T$ cell; exosome; extracellular vesicle; mesenchymal cell; tumor metastasis

ARTICLE INFO

Received: March 3, 2020

Accepted: April 1, 2020

Available online: April 14, 2020

*CORRESPONDING AUTHOR

Naohiro Seo, Department of Immuno-

Gene Therapy, Mie University

Graduate School of Medicine, 2-174,

Edobashi, Tsu, Mie 514-8507, Japan;

seo-naohiro@clin.medic.mie-u.ac.jp

\section{CITATION}

Seo N. Exosome-mediated immune regulation and its clinical application. Trends Immunother 2020; 4(1):36-41. doi: 10.24294/ti.v2.i1.433.

\section{COPYRIGHT}

Copyright (C) 2020 by author(s) and EnPress Publisher LLC. This work is licensed under the Creative Commons Attribution-NonCommercial 4.0 International License (CC BY-NC 4.0). http://creativecommons.org/licenses/ by $/ 4.0 /$

\section{Conventional immune system}

NK cells and macrophages are representative innate immune cells that act as a central player at an early stage of pathological conditions such as infection and cancer. In addition, extrathymically differentiated $\mathrm{T}$ cells expressing $\gamma \delta \mathrm{T}$ cell receptor (TCR), and NK T cells expressing NK makers and invariant $\alpha \beta$ TCR to $\alpha$-galactosylceramide ( $\alpha \mathrm{GalCer})$ in the context of CD1d are also classified as innate immune cells. On the other hand, intrathymic-differentiated T cells bore $\alpha \beta$ TCR can expand vigorously by priming with specific antigen peptide/major histocompatibility complex (MHC) on dendritic cells (DCs), exhibit effector/memory phenotype, and participate in the elimination of abnormal cells, so termed as acquired immune cells ${ }^{[1]}$. Acquired immunity is composed by $\mathrm{CD} 4^{+}$helper $\mathrm{T}$ (Th1 and $\mathrm{Th} 2$ ) cells, $\mathrm{CD} 4^{+} \mathrm{Foxp}^{+}$regulatory $\mathrm{T}$ (Treg) cells, and $\mathrm{CD} 8^{+}$cytotoxic $\mathrm{T}$ lymphocytes (CTLs). Interferon (IFN)- $\gamma$-expressing Th1 cells support CTL induction in antigenic stimulation by DCs. Interleukin (IL)-4 secreting Th2 cells promote $\mathrm{B}$ cell differentiation into antibody-producing plasma cells and tumorigenesis of epithelial cells, and inhibit inflammation. $\mathrm{CD}^{+}{ }^{+} \mathrm{Foxp}^{+}$Treg cells suppress antigen-specific $\mathrm{T}$ and $\mathrm{B}$ cell responses. In addition to Treg cells, myeloid-derived suppressor cells (MDSCs) strongly reduce T and NK cell activites and promote tumor infiltration of Treg cells ${ }^{[2]}$. Chemokines from macrophages and DCs attract innate immune cells and acquired immune cells in tumor lesion. It has been gradually clarified that exosomes released from these immunocompetent cells play a part of complicated immune responses ${ }^{[3]}$.

\section{Exosomes from $\mathrm{T}$ cells and NK cells}

$\mathrm{T}$ cells strongly release exosomes with activation ${ }^{[4]}$. Treg cell exosomes have been studied to some extent, all of which are reports regarding immunosuppressive function. CD73 on Treg cells converts extracellular ATP to immunosuppressive adenosine (ADO), and inhibits T cell and NK cell activities. Treg cell exosomes also express CD73 and seem to participate in the immunosuppression ${ }^{[5,6]}$. Treg cell exosomes inhibit strongly Th1 cell 
activity in an exosomal micro (mi) RNA-denpendent manner ${ }^{[7]}$. Transforming growth factor (TGF) $\beta$ and suppressive miRNAs in breast milk exosomes are retained relatively stable against temperature, $\mathrm{pH}$, and freeze-thaw, and they maintain Treg cells, and prevent the onset of modern diseases such as atopic dermatitis by reduction of IgE production of B cells $^{[8,9]}$. Suppressive role of Treg cell exosomes may be applicable in tolerance induction during organ transplantation $^{[10]}$.

$\mathrm{CD}^{+} \mathrm{T}$ cells proliferate predominantly when spleen cells of mouse and human peripheral blood lymphocytes are cultured by stimulation with both CD3 and CD28. It has been reported that exosomes from primary culture of $\mathrm{T}$ cells preferential activate naive $\mathrm{CD} 8^{+} \mathrm{T}$ cells, but not naive $\mathrm{CD} 4^{+} \mathrm{T}$ cells ${ }^{[11]}$. $\mathrm{CD}^{+} \mathrm{T}$ cells express FasL capable of apoptosis of $\mathrm{Fas}^{+}$tumor cells. However, FasL on $\mathrm{CD} 8^{+} \mathrm{T}$ cell exosomes seem to promote invasion and metastasis of tumor cells, but not tumor cell killing, by matrix metalloproteinase (MMP)-9-medaited degradation of extracellular matrix proteins via Fas/FasL signaling pathway $^{[12]}$. Conversely, since FasL was not found on $\mathrm{CD}^{+} \mathrm{T}$ cell exosomes in our study, we examined the action of murine $\mathrm{CD} 8^{+} \mathrm{T}$ cell exosomes in tumors in comparison with exosomes from other lymphocyte populations, tumor cells, or human $\mathrm{T}$ cells. Surprisingly, $\mathrm{CD} 8^{+} \mathrm{T}$ cell exosomes exhibit cytotoxicity against tumor stromal cells such as MSCs and CAFs, but not tumor cells. In addition, $\mathrm{CD}^{+} \mathrm{T}$ cell exosome-mediated destruction of mesenchymal stroma associated with the reduction of tumor invasion and metastasis (Figure 1) ${ }^{[13]}$.

Studies of NK cell exosomes have not progressed because of lack of explosive growth potential compared to $\mathrm{T}$ cells and difficulty of cultivation in a single population. NK cell exosomes express FasL, and seem to be able to induce apoptosis of Fas+ tumor cells. It has been reported that cytotoxic substances, perforin and granzyme B, are abundantly shown in the lumen of NK cell exosomes ${ }^{[14]}$.

\section{Relationship among B cells, macro- phage, and exosomes}

$\mathrm{B}$ cells and macrophages have been known as antigen-presenting cells. Major histocompatibility complex (MHC) class II molecules express on exosome membrane released from both cells ${ }^{[15]}$. B cell exosomes entering lymph node are rapidly taken up and decomposed by subcapsular macrophages. Interaction CD169 (Siglec-1) expressed on subcapsular macrophages with sialic acid on B cell exosomes is important in this setting ${ }^{[16]}$. It has been reported that exosomes from chronic B leukemia cells have a potential to differentiate vascular endothelial cells and MSCs into smooth muscle actin+ CAFs.

When exosomes are administered systemically, most of them are engulfed by hepatic macrophages and digested in the lysosome. Scavenger receptors

\section{$\mathrm{CD8}^{+} \mathrm{T}$ cell exosome}

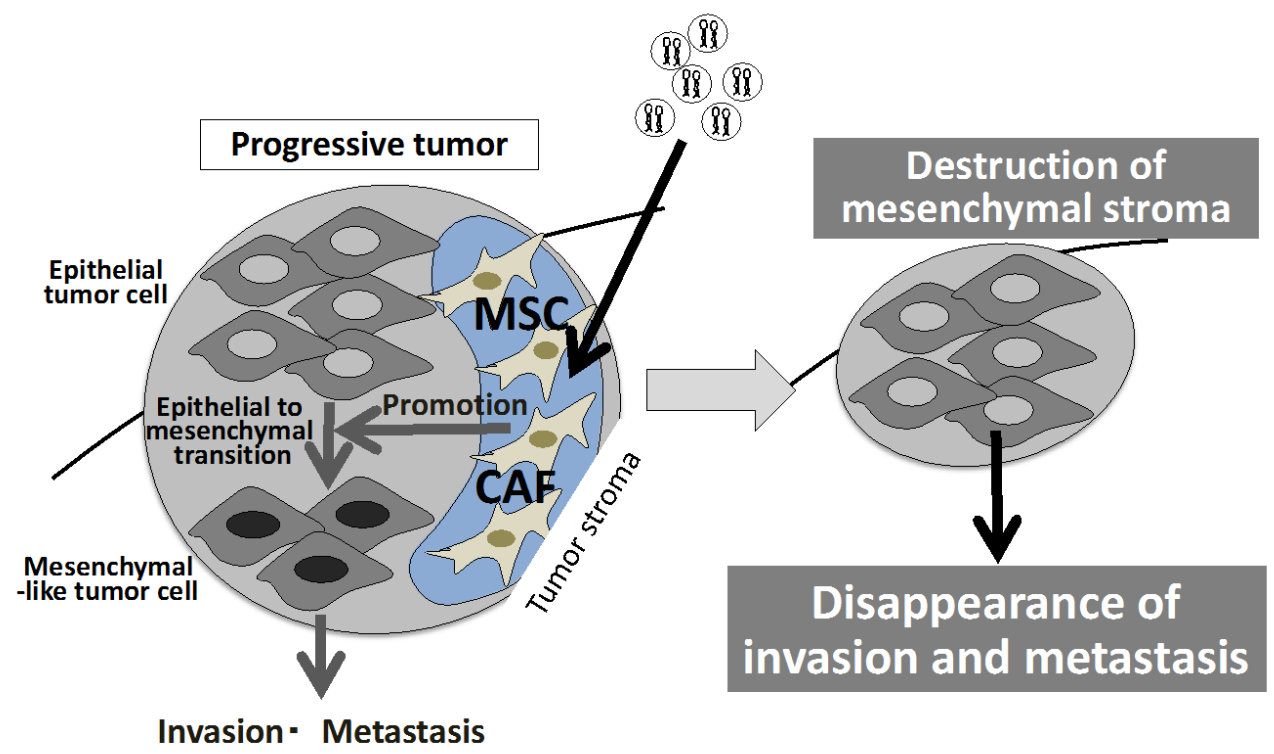

Figure 1. Prevention of tumor progression by $\mathrm{CD} 8+\mathrm{T}$ cell exosomes. $\mathrm{CD} 8^{+} \mathrm{T}$ cell exosomes deplete mesenchymal tumor stromal cells such as MSCs and CAFs. This exosome-mediated destruction of tumor stroma associates with reduction of tumor invasion and metastasis. 
such as SR-A (Scavenger receptor class A) on hepatic macrophages seem to be a ligand for phosphatidylserine-derived negative surface charge on exosomes ${ }^{[17,18]}$. In liver metastasis of pancreatic cancer, pancreatic cancer cell-released exosomes participate in the formation of pre-metastasis niche by promotion of TGF- $\beta$ and fibronectin production of hepatic stellate cells after kupffer cell activation ${ }^{[19]}$, showing close relationship between exosomes and liver macrophages. Exosomes released from tumor-associated macrophages seem to promote differentiation of monocytes into macrophages in an exosomal miRNA-dependent manner ${ }^{[20]}$.

\section{DC exosomes and immune regulation}

DCs reside in lymph nodes, skin and mucosal tissues in immature form. By capturing viral antigens or tumor proteins, immature DCs activate with enhanced expression of MHC class II molecules, migrate into draining lymph nodes, and present antigen peptides to specific $\mathrm{T}$ cells ${ }^{[21]}$. Immature DCs release exosomes more vigorously than mature DCs ${ }^{[22,23]}$. Immature DC exosomes exhibit a potent role in Treg cell activation ${ }^{[23,24]}$, suggesting maintenance of homeostasis by suppressing autoimmune reactions and excessive inflammations. Whereas, mature DCs release exosomes to facilitate tumoricidal immune reactions.
Hence DC exosomes pulsed with tumor antigen peptides can induce cytotoxic T lymphocyte (CTL) responses in $\mathrm{B}$ cell dependent manner (unknown mechanism) in vivo, clinical application of DC exosomes have already begun (Figure 2) ${ }^{[25-27]}$. However, DC exosomes seem to have high NK cell activating capacity rather than CTL induction ${ }^{[25,26,28]}$. DC exosomes pulsed with $\alpha$-galactosylceramide $(\alpha$-GalCer $)$ and tumor antigen peptides can activate invariant (i) NK T cells and $\gamma \delta \mathrm{T}$ cells concomitant with specific CTLs, resulting elicitation a strong antitumor immune responses ${ }^{[29]}$. MHC class I molecule on the exosome membrane exists as dimer at the end of the lumen region, and may have different conformation from that of the monomer ${ }^{[30]}$. Furthermore, exosomes derived from DCs from MHC class I knockout mice have T cell inducing and NK cell activating abilities comparable to those of DC exosomes from normal mice ${ }^{[31]}$, suggesting MHC class I-independent mechanism of antigen presentation by DC exosomes (Figure 2).

\section{Tumor cell exosomes and immune regulation}

Immune modulatory effect of tumor cell exosomes was most developed. Tumor cell-derived exosomes promote activation and accumulation of Treg cells ${ }^{[32-34]}$. Likewise, tumor cell exosomes enhance production of prostaglandin E2, IL6 , and TGF- $\beta$ of MDSCs, resulting formation of

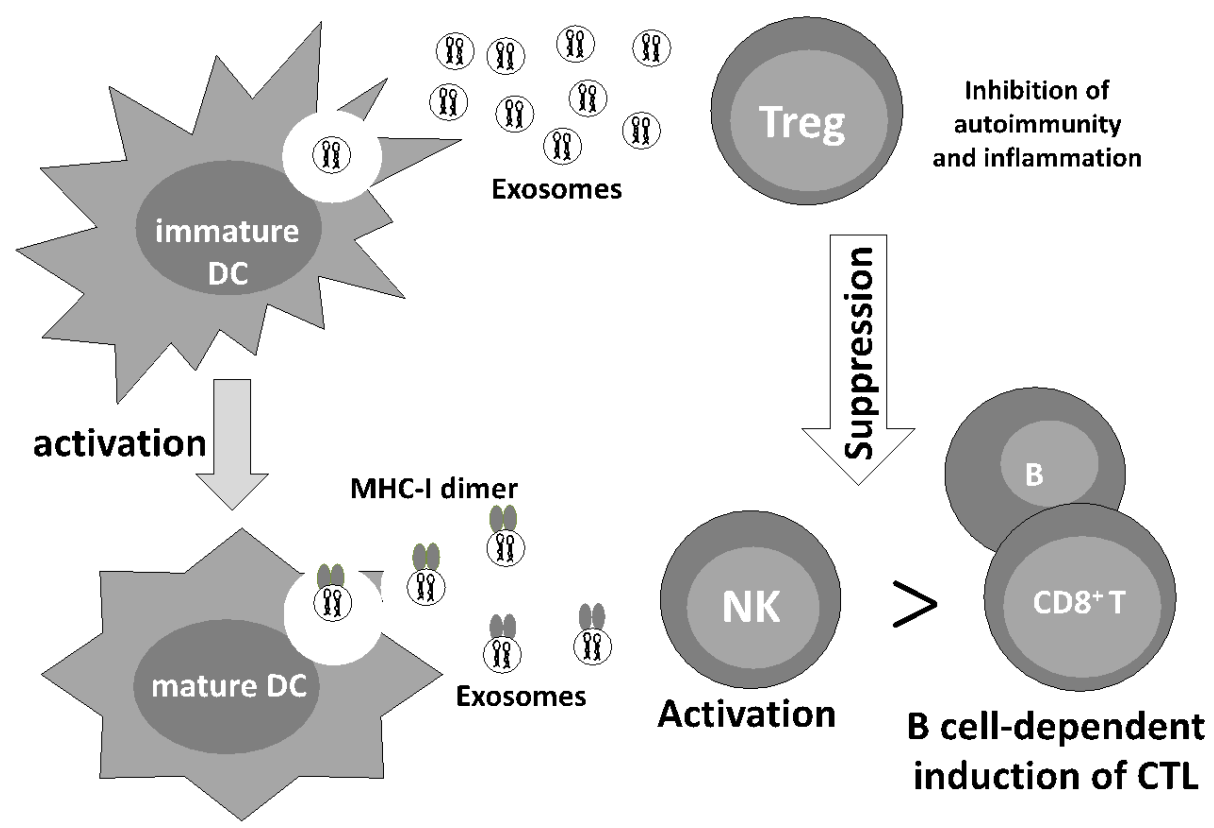

Figure 2. DC exosome-mediated activation of immune cells. Immature DCs and mature DCs release exosomes to induce Treg cells, and NK cells and CTLs, respectively. MHC class I molecules on exosomes from mature DCs express as a dimer. 
strong immunosuppressive environment in tumor lesions ${ }^{[35,36]}$. NK cells, $\gamma \delta$ T cells, and a part of CTLs recognize tumor surface MHC class I moleculelike UL16-binding protein (ULBP) ${ }^{[3]}$ and MHC class I polypeptide-related sequence A (MIC-A) by interaction with NKG2D, and can lyse tumor cells. However, tumor cells released ULBP- and MICA-bearing exosomes, and block cytotoxicity ${ }^{[37,38]}$. Interestingly, it has been reported that tumor cell exosome-engulfed DCs produce immune competent exosomes for effective induction of anti-tumor immunity ${ }^{[39,40]}$. This seems to be related to the Type-I IFN secretion mediated by cGAS (cyclic GMP-AMP synthase)/STING (Stimulation of IFN gene) pathway in DCs by exosomal DNAs ${ }^{[41]}$.

Tumor cells are always under hypoxic conditions and temperature stress, and are also exposed to drug stress during treatment of anticancer agents. Under these circumstances, it has been known that tumor cells release exosomes more aggressively than normal condition, and exhibit immune modulatory effects. In low oxigen, tumor cells released TGF- $\beta$ bearing exosomes, and promote and inhibit Treg cell activity and NK cell cytotoxicity, respectively ${ }^{[42]}$. Conversely, tumor cell exosomes released by high temperature stress or anticancer drug stress embed HSP (Heat shock protein)-70 and CCL (CC chemokine) $-2,-4,-5$, and -20 capable of promoting migration and activation of $\mathrm{T}$ cells, NK cells, and $\operatorname{DCs}^{[43,44]}$.

\section{Concluding remarks}

In immune system, exosomes seem to inherit the function of parent cells, implying exosomes from $\mathrm{CD} 8^{+} \mathrm{T}$ cells, macrophages, and $\mathrm{B}$ cells for treatment of tumors in addition to the already used DC exosomes. However, proteins expressed or embedded in exosomes may exhibit different function from those in the parent cells, as shown in MHC class I dimer on exosome membrane and MHC class I-independent activation of NK and CTLs by DC exosomes. Solving various issues including molecular structure should be important to elucidate the biological significance and clinical treatment of immune cell-derived exosomes.

\section{Conflict of interest}

The author declares no potential conflict of interest with respect to the research, authorship, and/ or publication of his article.

\section{References}

1. Seo N, Hayakawa S, Tokura Y. Mechanisms of immune privilege for tumor cells by regulatory cytokines produced by innate and acquired immune cells. Semin Cancer Biol 2002; 12(4): 291-300. doi: 10.1016/S1044-579X(02)00015-9.

2. Kumar V, Patel S, Tcyganov E, et al. The Nature of Myeloid-Derived Suppressor Cells in the Tumor Microenvironment. Trends Immunol 2016; 37(3): 208-220. doi: 10.1016/j.it.2016.01.004.

3. Gehrmann U, Näslund TI, Hiltbrunner S, et al. Harnessing the exosome-induced immune response for cancer immunotherapy. Semin Cancer Biol 2014; 28: 58-67. doi: 10.1016/ j.semcancer.2014.05.003.

4. Momose F, Seo N, Akahori Y, et al. Guanine-Rich Sequences Are a Dominant Feature of Exosomal microRNAs across the Mammalian Species and Cell Types. PLoS One 2016; 11(4): e0154134. doi: 10.1371/journal.pone.0154134.

5. Schuler PJ, Saze Z, Hong CS, et al. Human CD4+ CD39+ regulatory $\mathrm{T}$ cells produce adenosine upon co-expression of surface CD73 or contact with CD73+ exosomes or CD73+ cells. Clin Exp Immunol 2014; 177(2): 531-543. doi: 10.1111/ cei.12354.

6. Smyth LA, Ratnasothy K, Tsang JY, et al. CD73 expression on extracellular vesicles derived from CD4+ CD25+ Foxp3+ T cells contributes to their regulatory function. Eur J Immunol 2013; 43(9): 2430-2440. doi: 10.1002/eji.201242909.

7. Okoye IS, Coomes SM, Pelly VS, et al. MicroRNA-containing T-regulatory-cell-derived exosomes suppress pathogenic $\mathrm{T}$ helper 1 cells. Immunity 2014; 41(3): 89-103. doi: 10.1016/ j.immuni.2014.05.019.

8. Pieters BC, Arntz OJ1, Bennink MB, et al. Commercial cow milk contains physically stable extracellular vesicles expressing immunoregulatory TGF- $\beta$. PLoS One 2015; 10(3): e0121123. doi: 10.1371/journal.pone.0121123.

9. Melnik BC, John SM, Schmitz G. Milk: An exosomal microRNA transmitter promoting thymic regulatory $\mathrm{T}$ cell maturation preventing the development of atopy? J Transl Med 2014; 12: 43. doi: 10.1186/1479-5876-12-43.

10. Yu X, Huang C, Song B, et al. CD4+CD25+ regulatory $\mathrm{T}$ cells-derived exosomes prolonged kidney allograft survival in a rat model. Cell Immunol 2013; 285: 62-68. doi: 10.1016/ j.cellimm.2013.06.010.

11. Wahlgren J, Karlson Tde L, Glader P, et al. Activated human $\mathrm{T}$ cells secrete exosomes that participate in IL-2 mediated immune response signaling. PLoS One 2012; 7(11): e49723. doi: 10.1371/journal.pone.0049723. 
12. Cai Z, Yang F, Yu L, et al. Activated T cell exosomes promote tumor invasion via Fas signaling pathway. J Immunol 2012; 188(12): 5954-5961. doi: 10.4049/jimmunol.1103466.

13. Seo N, Shirakura Y, Tahara Y, et al. Activated CD8+ T cell extracellular vesicles prevent tumour progression by targeting of lesional mesenchyal cells. Nat Commun 2018; 9(1): 435. doi: 10.1038/ s41467-018-02865-1.

14. Lugini L, Cecchetti S, Huber V, et al. Immune surveillance properties of human NK cell-derived exosomes. J Immunol 2012; 189(6): 2833-2842. doi: 10.4049/jimmunol.1101988.

15. Raposo G, Nijman HW, Stoorvogel W, et al. B lymphocytes secrete antigen-presenting vesicles. J Exp Med 1996; 183(3): 1161-1172. doi: 10.1084/ jem.183.3.1161.

16. Saunderson SC, Dunn AC, Crocker PR, et al. CD169 mediates the capture of exosomes in spleen and lymph node. Blood 2014; 123(2): 208-216. doi: 10.1182/blood-2013-03-489732.

17. Paggetti J, Haderk F, Seiffert M, et al. Exosomes released by chronic lymphocytic leukemia cells induce the transition of stromal cells into cancerassociated fibroblasts. Blood 2015; 126(9): 11061117. doi: 10.1182/blood-2014-12-618025.

18. Matsumoto A, Takahashi Y, Nishikawa M, et al. Role of Phosphatidylserine-Derived Negative Surface Charges in the Recognition and Uptake of Intravenously Injected B16BL6-Derived Exosomes by Macrophages. J Pharm Sci 2017; 106(1): 168175. doi: 10.1016/j.xphs.2016.07.022.

19. Costa-Silva B, Aiello NM, Ocean AJ, et al. Pancreatic cancer exosomes initiate pre-metastatic niche formation in the liver. Nat Cell Biol 2015; 17(6): 816-826. doi: 10.1038/ncb3169.

20. Ismail N, Wang Y, Dakhlallah D, et al. Macrophage microvesicles induce macrophage differentiation and miR-223 transfer. Blood 2013; 121(6): 984 995. doi: 10.1182/blood-2011-08-374793.

21. Seo N, et al. Basic and clinical aspects of epidermal Langerhans cell-based tumor immunotherapy. Expert Rev Dermatol 2007; 2: 725-733. doi: 10.1586/17469872.2.6.725.

22. Quah BJ, O'Neill HC. The immunogenicity of dendritic cell-derived exosomes. Blood Cells Mol Dis 2005; 35(2): 94-110. doi: 10.1016/ j.bcmd.2005.05.002.

23. Quah BJ, O'Neill HC. Maturation of function in dendritic cells for tolerance and immunity. J Cell Mol Med 9: 643-654, 2005. doi: 10.1111/j.15824934.2005.tb00494.x.

24. Li X, Li JJ, Yang JY, et al. Tolerance induction by exosomes from immature dendritic cells and rapamycin in a mouse cardiac allograft model. PLoS One 2012; 7(8): e44045. doi: 10.1371/ journal.pone. 0044045 .
25. Pitt JM, André F, Amigorena S, et al. Dendritic cell-derived exosomes for cancer therapy. J Clin Invest 2016; 126(4): 1224-1232. doi: 10.1172/ JCI81137.

26. Gehrmann U, Näslund TI, Hiltbrunner S, et al. Harnessing the exosome-induced immune response for cancer immunotherapy. Semin Cancer Biol 2014; 28: 58-67. doi: 10.1016/ j.semcancer.2014.05.003.

27. Näslund TI, Gehrmann U, Qazi KR, et al. Dendritic cell-derived exosomes need to activate both $\mathrm{T}$ and $\mathrm{B}$ cells to induce antitumor immunity. $\mathrm{J}$ Immunol 2013; 190(6): 2712-2719. doi: 10.4049/ jimmunol.1203082.

28. Besse B, Charrier M, Lapierre V, et al. Dendritic cell-derived exosomes as maintenance immunotherapy after first line chemotherapy in NSCLC. Oncoimmunology 2015; 5(4): e1071008. doi: 10.1080/2162402X.2015.1071008.

29. Gehrmann U, Hiltbrunner S, Georgoudaki $\mathrm{AM}$, et al. Synergistic induction of adaptive antitumor immunity by codelivery of antigen with $\alpha$-galactosylceramide on exosomes. Cancer Res 2013; 73(13): 3865-3876. doi: 10.1158/0008-5472. CAN-12-3918.

30. Lynch S, Santos SG, Campbell EC, et al. Novel MHC class I structures on exosomes. J Immunol 2009; 183(3): 1884-1891. doi: 10.4049/ jimmunol.0900798.

31. Hiltbrunner S, Larssen P, Eldh M, et al. Exosomal cancer immunotherapy is independent of MHC molecules on exosomes. Oncotarget 2016; 7(25): 38707-38717. doi: 10.18632/oncotarget.9585.

32. Muller L, Mitsuhashi M, Simms P, et al. Tumorderived exosomes regulate expression of immune function-related genes in human T cell subsets. Sci Rep 2016; 6: 20254. doi: 10.1038/srep20254.

33. Mrizak D, Martin N, Barjon C, et al. Effect of nasopharyngeal carcinoma-derived exosomes on human regulatory T cells. J Natl Cancer Inst 2014; 107(1): 363. doi: 10.1093/jnci/dju363.

34. Szajnik M, Czystowska M, Szczepanski MJ, et al. Tumor-derived microvesicles induce, expand and up-regulate biological activities of human regulatory $\mathrm{T}$ cells (Treg). PLoS One 2010; 5(7): e11469. doi: 10.1371/journal.pone.0011469.

35. Chalmin F, Ladoire S, Mignot G, et al. Membraneassociated Hsp72 from tumor-derived exosomes mediates STAT3-dependent immunosuppressive function of mouse and human myeloid-derived suppressor cells. J Clin Invest 2010; 120(2): 457471. doi: 10.1172/JCI40483.

36. Xiang X, Poliakov A, Liu C, et al. Induction of myeloid-derived suppressor cells by tumor exosomes. Int J Cancer 2009; 124(11): 2621-2633. doi: $10.1002 /$ ijc. 24249 .

37. Fernández-Messina L, Ashiru $\mathrm{O}$, Boutet $\mathrm{P}$, et al. Differential mechanisms of shedding of the 
glycosylphosphatidylinositol (GPI)-anchored NKG2D ligands. J Biol Chem 2010; 285(12): 8543-8551. doi: /10.1074/jbc.M109.045906.

38. Ashiru O, Boutet P, Fernández-Messina L, et al. Natural killer cell cytotoxicity is suppressed by exposure to the human NKG2D ligand MICA*008 that is shed by tumor cells in exosomes. Cancer Res 2010; 70(2): 481-489. doi: 10.1158/00085472.CAN-09-1688.

39. 39. Bu N, Wu H, Sun B, et al. Exosome-loaded dendritic cells elicit tumor-specific CD8+ cytotoxic $\mathrm{T}$ cells in patients with glioma. J Neurooncol 2011; 104(3): 659-667. doi: 10.1007/s11060-011$0537-1$.

40. Yang N, Li S, Li G, et al. The role of extracellular vesicles in mediating progression, metastasis and potential treatment of hepatocellular carcinoma. Oncotarget 2017; 8(2): 3683-3695. doi: 10.18632/ oncotarget. 12465.

41. Zhang H, Tang K, Zhang Y, et al. Cell-free tumor microparticle vaccines stimulate dendritic cells via cGAS/STING signaling. Cancer Immunol Res 2015; 3(2): 196-205. doi: 10.1158/2326-6066.CIR14-0177.

42. Berchem G, Noman MZ, Bosseler M, et al. Hypoxic tumor-derived microvesicles negatively regulate NK cell function by a mechanism involving TGF- $\beta$ and miR23a transfer. Oncoimmunology 2015; 5(4): e1062968. doi: 10.1080/2162402X.2015.1062968.

43. Chen T, Guo J, Yang M, et al. Chemokinecontaining exosomes are released from heatstressed tumor cells via lipid raft-dependent pathway and act as efficient tumor vaccine. $\mathrm{J}$ Immunol 2011; 186(4): 2219-2228. doi: 10.4049/ jimmunol.1002991.

44. Lv LH, Wan YL, Lin Y, et al. Anticancer drugs cause release of exosomes with heat shock proteins from human hepatocellular carcinoma cells that elicit effective natural killer cell antitumor responses in vitro. J Biol Chem 2012; 287(19): 15874-15885. doi: 10.1074/jbc.M112.340588. 